\title{
Mucormycosis in a patient with acute myeloblastic leukemia following liver transplantation for Wilson's disease
}

\author{
Aleksandra Anna Łanocha ${ }^{1, A, C-D \oplus}$, Renata Guzicka-Kazimierczak ${ }^{1, B-C \oplus}$, Barbara Zdziarska $^{1, B-C \oplus}$, \\ Marta Wawrzynowicz-Syczewska, ${ }^{1, \mathrm{~B}-\mathrm{C} \oplus}$ \\ ${ }^{1}$ Pomeranian Medical University, Szczecin, Poland \\ A - Research concept and design, B - Collection and/or assembly of data, C - Data analysis and interpretation, \\ $D$ - Writing the article, $E$ - Critical revision of the article, $F$ - Final approval of article
}

Łanocha A. A, Guzicka-Kazimierczak R, Zdziarska B, Wawrzynowicz-Syczewska M. Mucormycosis in a patient with acute myeloblastic leukemia following liver transplantation for Wilson's disease. Ann Agric Environ Med. 2019; 26(4): 665-668. doi: 10.26444/aaem/105533

\begin{abstract}
A case is presented of mucormycosis in a patient with acute myeloblastic leukemia following liver transplantation for Wilson's disease. A 58-year-old female was admitted to the Department of Haematology with deterioration of her general condition, loss of appetite, tiredness and difficulty with mental contact for a few days. Blood and urine cultures for bacteria and fungus, galactomannan antigen were negative. Whole body computed tomography demonstrated bilateral hilar lymphadenopathy with necrotic lesions: splenomegaly with a hypodensive lesion $13 \times 20 \times 19 \mathrm{~mm}$ and lower pulmonary infiltrates suggested fungal etiology. Magnetic resonance imaging of the brain showed thickened meninges. Finally, mucormycosis was diagnosed. Treatment with amphotericin B lipid complex was started, resulting in an partial improvement of the general condition and decreased level of inflammatory markers. However, the patient's condition continued to deteriorate, with sepsis etiology Escherichia coli, and despite the intensive managements she eventually died.
\end{abstract}

Key words

Mucormycosis, acute myeloblastic leukemia, Willson's disease, liver transplantation

\section{INTRODUCTION}

Mucormycosis (zygomycosis) is a rare but still fatal opportunistic invasive fungal infection caused by filamentous fungi from the orders of Morales and Entomophthorales [1]. The incidence of invasive fungal infections, (IFI) has increased in recent years as a result of severe and long-lasting immunosupression and neutropenia [2]. After aspergillosis and candidiasis, mucormycosis is the third most common cause of IFI in patients with haematologic malignancies after chemotherapy and solid organ transplantation [3]. Mechanisms of disease are related to conspicuous deficient action of polymorphonuclear and mononuclear phagocytes [4]. Data shows an increasing incidence of mucormycosis from $0.7 /$ million in 1997 to $1.2 /$ million in 2006, and in patients with haematologic malignancies from $0.02 /$ million to 0.2 /million [5]. Sarvestani et al. [6] observed that the prevalence rate of mucormycosis infection was generally 4.27 per 100 patients with haematologic malignancies. The clinical and radiological manifestations of mucormycosis are unspecific. Diagnosis and routine investigation includes also microbiological, histological and cytological findings [7].

Wilson's disease (WD) is an inherited disorder of copper metabolism with a wide spectrum of clinical symptoms, mainly hepatic or neurological. Liver transplantation may correct the underlying hepatic metabolic defect and is indicated for the $\sim 5 \%$ of WD patients with acute failure as the first presentation of disease [8]. A case is presented of mucormycosis in a patient with acute myeloblastic leukemia (AML) following liver transplantation for WD.

Address for correspondence: Aleksandra Anna Łanocha, Pomeranian Medical University in Szczecin, Poland

e-mail: aleksandra.lanocha@pum.edu.pl

Received: 11.01.2019; accepted: 12.02.2019; first published: 18.03.2019

\section{CASE REPORT}

In 2001, a 58-year-old female was diagnosed with WD (hepatic neurological type) and in 2012 underwent liver transplantation. In April 2015, after reconstruction of the arterial anastomosis of the transplanted liver due to hepatic artery stenosis and cholecystectomy, the patient was diagnosed with acute myeloblastic leukemia FLT3(-), NPM1(-), CEBPA(-). She was treated with chemotherapy according to the Polish Adults Leukemia Group (PALG): induction of DAC (daunorubicine, cytarabine and cladribine) $<60$-year-old protocol, followed by reinduction chemotherapy CLAG-M (cladribine, cytarabine, mitoxantron and GCS-F). Antifungal prophylaxis with posaconazole was used. On day 30 , after the $2^{\text {nd }}$ cycle of chemotherapy, pancytopenia was present and aplastic anaemia was diagnosed in bone marrow. Oral administration of a low dose of methylprednisolone was therefore was started. The patient was admitted to the Department of Haematology with deterioration of her general condition, loss of appetite, tiredness and difficulty with mental contact for a few days. On physical examination, the patient was unconscious, dehydrated, with petechiae, haematoma on the dorsum of the nose and tachycardia. A blood test revealed pancytopenia with a white blood cell count of $0.44 \mathrm{G} / \mathrm{l}$, platelet count $-1.0 \mathrm{G} / \mathrm{l}$ and agranulocytosis - $0.06 \mathrm{G} / \mathrm{l}$, an increased level of inflammatory markers (CRP-115.7mg/l; prokalcytonine2,74ng/ml), hyperglycaemia $483 \mathrm{mg} / \mathrm{dl}$, ketoacidosis, increased aminotranspherase (ALT: $187 \mathrm{U} / \mathrm{l}, \mathrm{AST}: 305 \mathrm{U} / \mathrm{l})$, increased GGTP: $98 \mathrm{U} / \mathrm{l}$ and lactic dehydrogenase- $966 \mathrm{U} / \mathrm{l}$, normal total bilirubin $0.40 \mathrm{mg} / \mathrm{dl}$, creatinine $1.00 \mathrm{mg} / \mathrm{dl}$.

In the body computed tomography of the head, thickening of the mucosa of the maxillary sinus (greater on the right), ethmoid cells, frontal and sphenoid sinus were described. 
Blood and urine cultures for bacteria and fungus, and galactomannan antigen were negative. The patient's diabetic state was controlled with insulin and widespectrum antibacterial therapy (ceftriaxone, amikacin) was initiated. On the days 2 and 3 of the hospital stay, the patient developed a fever; meropenem, vancomycine, caspofungine were started. Aspergillosis was suspected and a whole body computed tomography was therefore performed and treatment with voriconazole administered. The whole body CT demonstrated bilateral hilar lymphadenopathy with necrotic lesions: left side $-29 \times 23 \times 25 \mathrm{~mm}$ and right side $-21 \times 14 \times 13 \mathrm{~mm}$, multiple nodules, parenchymal density and pleural effusions, splenomegaly with a hypodensive lesion measuring $13 \times 20 \times 19 \mathrm{~mm}$, and lower pulmonary infiltrates, suggested fungal etiology (Fig. 1 and 2). Magnetic resonance imaging of the brain showed thickened meninges (Fig.3). Cultures from cerebrospinal fluid were negative; cerebrospinal fluid analysis excluded myeloblasts.

During hospitalization, necrotic eschar was present on the dorsum of the nose. Scrapings for microbiological tests were therefore taken which showed Rhizomucor. Control blood and urine cultures were positive for Candida glabrata in the urine. In the treatment, voriconasole was changed to amphotericin B lipid complex (on day 10 of hospitalization), resulting in a partial improvement in the patient's general condition, and a decreased level of inflammatory markers. However, her condition continued to deteriorate, with sepsis etiology Escherichia coli, and despite the intensive managements she eventually died.
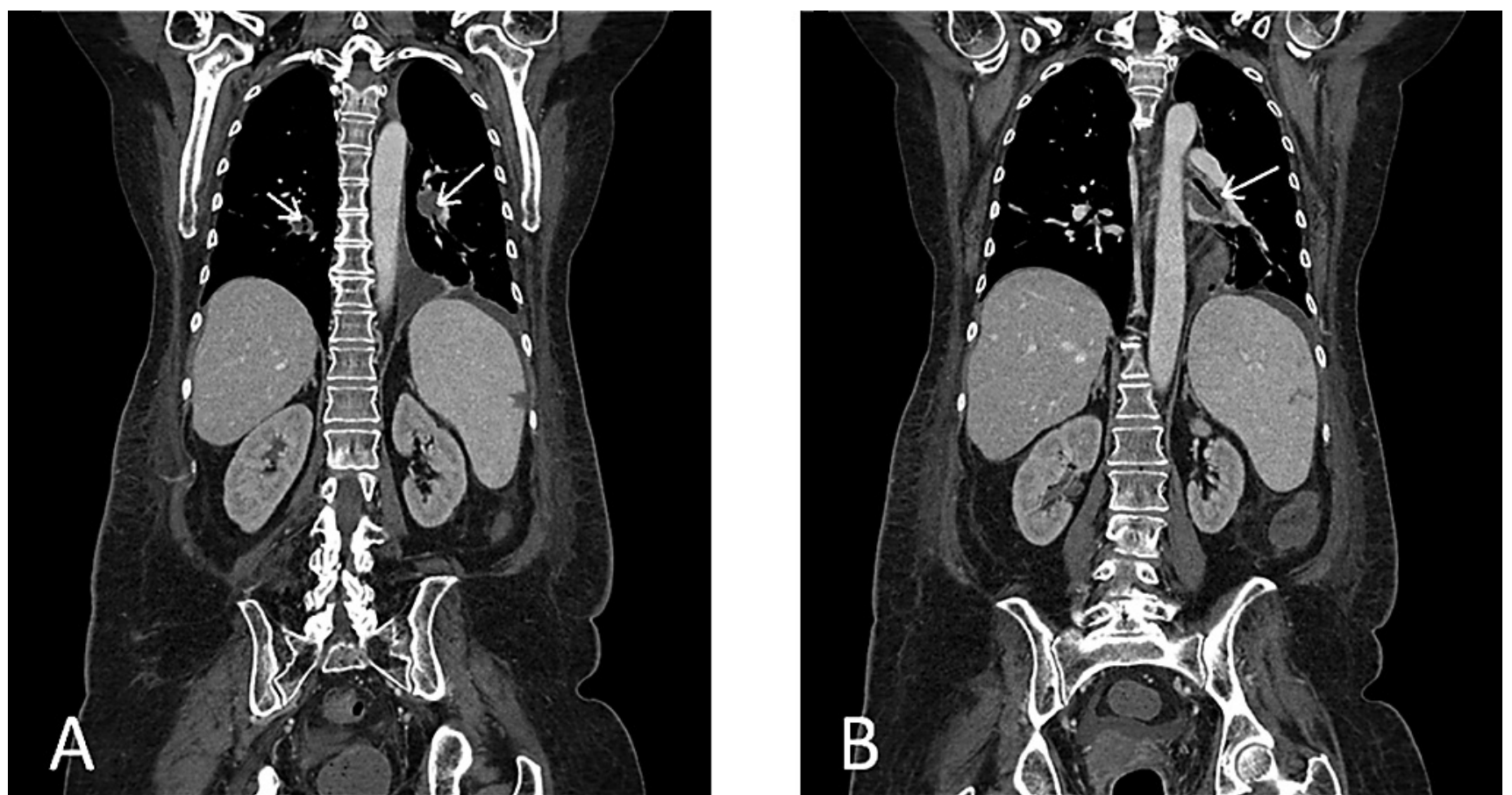

Figure 1. Chest and abdomen computed tomography showed bilateral hilar lymphadenopathy with necrotic lesions: $29 \times 23 \times 25 \mathrm{~mm}(\mathrm{~A}) ; 21 \times 14 \times 13 \mathrm{~mm}$, multiple nodules, parenchymal density and pleural effusions, splenomegaly with a hypodensive lesion $13 \times 20 \times 19 \mathrm{~mm}$ (B)

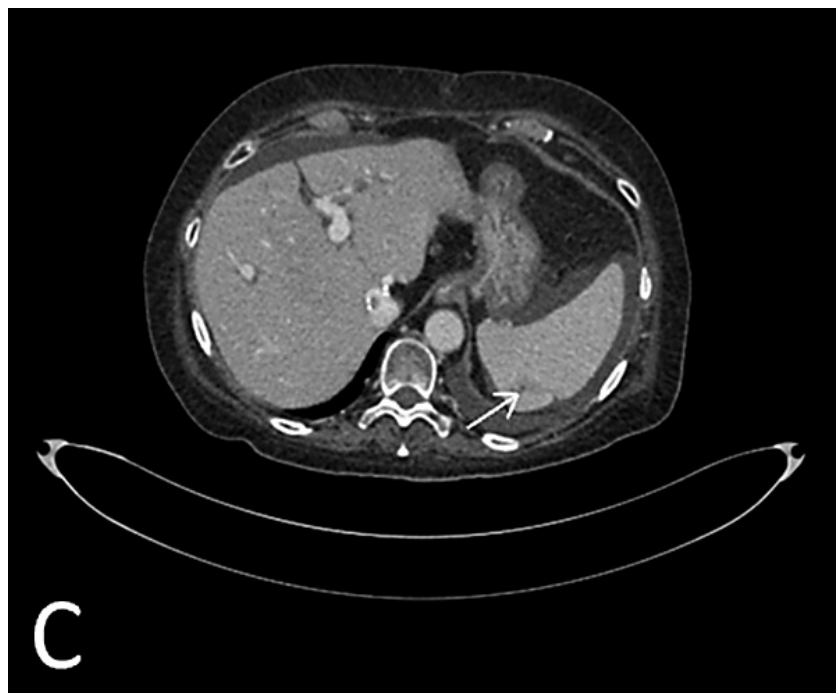

Figure 2. Chest and abdomen computed tomography showed splenomegaly with a hypodensive lesion $13 \times 20 \times 19 \mathrm{~mm}$ (C)

\section{DISCUSSION}

Mucormycosis is an uncommon and life-threatening, fungal infection caused by Mucorales spp. [3]. Risk factors are diabetes, acidosis, steroid use, haematologic malignancy, transplantation, severe trauma, burns, neutropenia and renal failure $[9,10]$. Mucormycosis can be classified as one of six forms: rhinocerebral, pulmonary, cutaneous, gastrointestinal, disseminated and uncommon presentations [11]. Patients with leukaemia have traditionally been considered to represent the majority of cases with pulmonary mucormycosis [12]. In the pulmonary or sinus form, exposure occurs by inhaling aerosolized mucor spores from the environment. Gastrointestinal mucormycosis, resulting from ingestion of spore-contaminated food, and cutaneous mucormycosis (very rare cases) due to direct skin implantation of fungal spores [2]. The patient in the presented case had the mentioned key predisposing factors: diabetes, neutropenia, acute myeloblastic leukaemia, chemotherapy, immunosupression after liver transplantation, treated with steroids. The clinical 

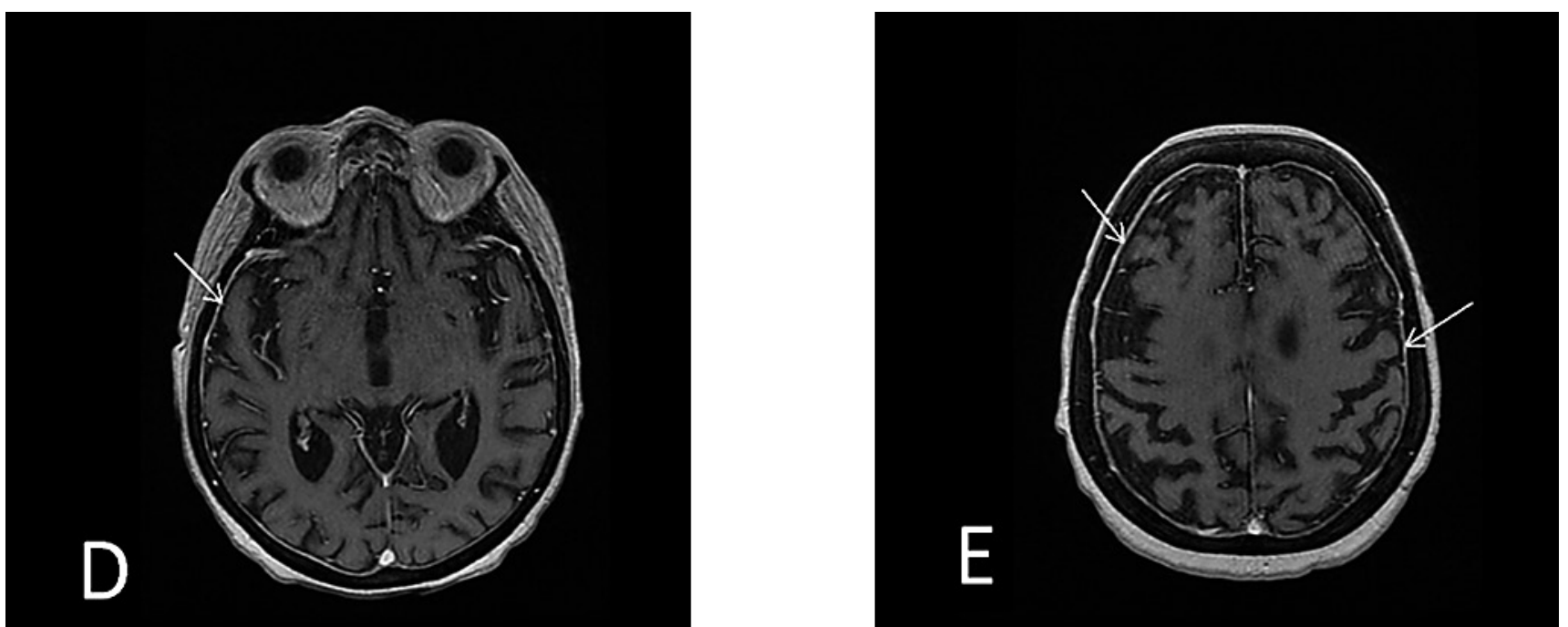

Figure 3. Magnetic resonance imaging of the brain showed thickening of the meninges ( $D$ and $E$ )

signs and symptoms of mucormycosis are nonspecific. There are no biomarkers to identify disease. $\beta$-D-glucan test and Aspergillus galactomannan tests do not detect antigen of the Mucorales cell wall [13]. Identification of mucormycosis is very difficult, most often comes from microscopic, culture and/or histopathological examination of relevant samples. To diagnose mucormycosis and assess disease staging, extensive clinical examination for signs dissemination, CT of brain, sinuses and chest, bronchoscopy with bronchoalveolar lavage and biopsy suspicious lesions of hard palette, skin, sinuses necessary [13]. New diagnostic approaches contain molecular testing and various other clinical samples including formalinfixed tissues (MALDI-TOF) and Mucorales-specific T-cell detection $[14,15]$. Unfortunately, molecular procedures are not widely available and diagnosis is based on fungal isolation and/or direct examination in clinical samples. Management includes association between antifungal, surgery and control of diabetes, metabolic abnormalities, such as haemochromatosis, haematopoietic growth factor of neutropenia, discontinuation/tapering of steroids and reduction of immunosuppressive therapy. Small focal lesions of mucormycosis should be surgically resected. Amphotericin $\mathrm{B}$, posaconazole, isavuconazole are the most potent agents. Echinocandins in association with L-AmB; L-AmB associated with posaconazole can be used in salvage therapy $[13,14]$. Amphotericin B is considered the drug of choice for primary treatment of mucormycosis. The standard daily dose of liposomal amphotericin B and lipid complex amphotericin $\mathrm{B}$ is $5 \mathrm{mg} / \mathrm{kg} /$ day. Although dosages beyond $5 \mathrm{mg} / \mathrm{kg} /$ day may be considered, for example when there is central nervous system or osteoarticular involvement [10]. Posaconazole may be considered as salvage treatment of mucormycosis or as a first-line treatment only in cases when amphotericin B treatment is absolutely contraindicated. Isavuconazole is a new broad-spectrum triazole, used for the treatment of mucormycosis when amphotericin B is not feasible. In the presented case, aspergillosis was initially suspected, therefore voriconazole was administered. On day 10 of hospitalization, voriconazole was changed to amphotericin B lipid complex, resulting in an partial improvement of the general condition and decreased level of inflammatory markers.

Mucormycosis still remains a fatal prognosis, with high mortality rates, ranking from $20-50 \%$ if localised, up to
$70-90 \%$ in cases of disseminated disease $[10,16]$. To the best of the authors' knowledge, this is the first described case of mucormycosis in a patient with WD after liver transplantation and acute myeloblastic leukaemia.

The presented case illustrates the dramatic course of mucormycosis and confirms the fatal prognosis associated with high mortality. The lack of availability of fast and reliable diagnostic tests means that it still remains a problem. The management of mucormycosis, including antifungal therapy, surgery and control of underlying conditions, should be started as soon as possible.

\section{Acknowledgements}

The authors would like to thank Dr Ewa Gabrysz-Trybek for assistance with preparation of the Figures.

\section{Conflicts of Interest}

The authors declare no conflict of interest.

\section{Ethical Approval}

No approval was required.

\section{Funding Source}

The research did not receive any specific grant from funding agencies in the public, commercial, or not-for-profit sectors.

\section{REFERENCES}

1. Kwon-Chung KJ. Taxonomy of fungi causing mucormycosis and entomophthoramycosis (zygomycosis) and nomenclature of the disease: molecular mycologic perspectives. Clin Infect Dis. 2012; 54: S8-S15.

2. Serio B, Rosamillo R, Giuduce V, Zeppa P, Esposito S, Fontana R, Annunziata S, Selleri C. Successful management of pulmonary mucormycosis with liposomal amphotericin B and surgery treatment: a case report. Infez Med. 2012; 2: 43-47.

3. Stefaniak MJ. Mucormycosis- Clinical manifestations, diagnosis and management. Zakażenia XXI wieku 2018; 1(1): 1-9.

4. Täger FM, Zaror CL, Martínez DP. Cutaneous mucormycosis in an immunocompromised patient. Rev Chilena Infectol. 2012; 29:101-107.

5. Bitar D, Van Cauteren D, Lanternier F, Dannaoui E, Che D, Dromer F. et al. Increasing Incidence of Zygomycosis (Mucormycosis), France, 1997-2006. Emerg Infect Dis. 2009; 15(9): 1395-1401.

6. Sarvestani A, Pishdad G, Bolandparvaz S. Epidemiology and Clinical Characteristics of Mucormycosis in Patients with Leukemia; A 21-year Experience from Southern Iran. Bull Emerge Trauma. 2014; 2(1): 38-43. 
7. Ribeiro EF, dos Santos VM, Paixão GT, Cruz LR, Danilow MZ, Campos VF. Mucormycosis in a patient with acute myeloid leukemia successfully treated with liposomal amphotericin B associated with deferasirox and hyperbaric oxygen. Mycopathologia. 2013; 175(3-4): 295-300.

8. Ahmad A, Torrazza-Perez E, Schilsky ML. Liver transplantation for Wilson disease. Handb Clin Neurol. 2017; 142: 193-204.

9. Clerigo V, Fernandes L, Abreu T, Barradas P. A rare case of pulmonary mucormycosis successfully treated with dual antifungal agents and surgery in patient with uncontrolled diabetes. Open J Respir Dis. 2016; 6: 35-40.

10. Sipsas NV, Gamaletsou MN, Anastasopoulou A, Kontoyiannis DP. J Fungi. 2018; 4(90): 1-17.

11. Petricos G, Skiada A, Lortholary O, Roilides E, Walsh TJ, Kontoyiannis DP. Epidemiology and clinical manifestation of Mucormycosis. Clin Infect Dis. 2012; 54(S1): S23-24.

12. Chakrabrati A, Das A, Sharma A, Panda N, Das S, Gupta KL, Sakhuja V. Ten years' experience in zygomycosis at a tertiary care center in India. J Infect. 2001; 42(4): 261-6.
13. Kontoyiannis DP, Lewis RE. How I treat mucormycosis. Blood. 2011; 118: $1216-1224$

14. Tissot F, Agrawal S, Pagano L, Petrikkos G, Groll AH, Skiada A, LassFlörl C, Calandra T, Viscoli C, Herbrecht R. ECIL- 6 guidelines for the treatment of invasive candidiasis, aspergillosis and mucormycosis in leukemia and hematopoietic stem cell transplant patients. Haematologica. 2017; 102: 433-444.

15. Millon L, Herbrecht R, Grenouillet F, Morio F, Alanio A, Letscher-Bru V, Cassaing S, Chouaki T, Kauffmann-Lacroix C, Poirier P, Toubas D, Augereau O, Rocchi S, Garcia-Hermoso D, Bretagne S; French Mycosis Study Group. Early diagnosis and monitoring of mucormycosis by detection of circulating DNA in serum: retrospective analysis of 44 cases collected through the French Surveillance Network of Invasive Fungal Infections (RESSIF). Clin Microbiol Infect. 2016; 22: 810.e1-810. e8.

16. Starkey J, Moritani T, Kirby P. MRI of CNS fungal infections: review of aspergillosis to histoplasmosis and everything in between. Clin Neuroradiol. 2014; 24:217-230.

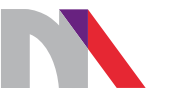

Ministry of Science and Higher Education

Republic of Poland

Generation of the DOI (Digital Object Identifier) - task financed under the agreement No. 618/P-DUN/2019 by the Minister of Science and Higher Education 